\title{
Weights and conservativity
}

\author{
Jörg Wildeshaus
}

\begin{abstract}
The purpose of this article is to study conservativity in the context of triangulated categories equipped with a weight structure. As an application, we establish (weight) conservativity for the restriction of the (generic) $\ell$-adic realization to the category of motives of Abelian type of characteristic zero.
\end{abstract}

\section{Introduction}

The aim of this article is to provide a proof of the weight conservativity of the restriction of the (generic) $\ell$-adic realization $R_{\ell}$ to the category of motives of Abelian type of characteristic zero (Theorem 4.4).

Here, weight conservativity refers to the following refinement of the usual categorical notion of conservativity: the functor $R_{\ell}$ detects not only isomorphisms but also weights (and hence, their absence). In other words, a motive $M$ of Abelian type is without weights $\alpha, \alpha+1, \ldots, \beta$, for integers $\alpha \leqslant \beta$, in the sense of [Wil09, Definition 1.10], if and only if the same is true for $R_{\ell}(M)$. This provides the main motivation for our study: as shown in op. cit., the absence of certain weights in the boundary motive of a scheme $X$ allows for the construction of its interior motive.

Weight conservativity for motives of Abelian type over a point was previously established in [Wil15, Section 1]. This special case suffices, for example, for the analysis of weights in the boundary motive of Picard surfaces [Wil15, Section 3], essentially because the complement of the latter in their Baily-Borel compactifications is of dimension zero. The study of the boundary motive of Shimura varieties, whose boundary is higher-dimensional, requires a version of weight conservativity for motives over higher-dimensional bases, whence the need for Theorem 4.4. For an application to the case of Siegel threefolds, we refer to [Wil17b].

In order to prove Theorem 4.4 and the intermediate conservativity results leading up to it (Theorems 3.11 and 4.3), it turns out that the notion of weight structure [Bon10] is central. In fact, the formal structure of the proofs is best understood in that abstract setting. This explains the title of the present work and also its organization. Section 2 is entirely situated in the context of functors $r$ whose source is a triangulated category $\mathcal{C}$ equipped with a weight structure $w$. The following question appears natural: assuming that the weight structure is bounded and $r$ is weight exact, does conservativity of the restriction of $r$ to the heart $\mathcal{C}_{w=0}$ imply (weight)

Received 20 September 2015, accepted in final form 12 July 2017.

2010 Mathematics Subject Classification 14F20, 14C15, 14C25, 14F25, 14F42, 18A22, $19 \mathrm{E} 15$.

Keywords: weight structures, conservativity, weight conservativity, realizations, relative motives of Abelian type. This journal is (C) Foundation Compositio Mathematica 2018. This article is distributed with Open Access under the terms of the Creative Commons Attribution Non-Commercial License, which permits non-commercial reuse, distribution, and reproduction in any medium, provided that the original work is properly cited. For commercial re-use, please contact the Foundation Compositio Mathematica.

Partially supported by the Agence Nationale de la Recherche, project "Régulateurs et formules explicites". 


\section{WEIGHTS AND CONSERVATIVITY}

conservativity of $r$ ? The analogous question for $t$-structures can be asked; there, the answer is obviously positive. Weight structures being much less rigid than $t$-structures, it is not surprising to see that additional hypotheses are needed. The main such condition concerns the heart: all our abstract results (Theorems 2.5, 2.8, 2.10, and 2.11) require $\mathcal{C}_{w=0}$ to be semi-primary [AK02, Définition 2.3.1]. The reason is that all proofs make systematic use of the minimal weight filtration [Wil17a, Section 2], whose existence is guaranteed only if $\mathcal{C}_{w=0}$ is semi-primary.

This explains why we restrict our attention to motives of Abelian type: while relative Chow motives may always be expected to form a semi-primary category [Wil17a, Conjecture 3.4], we are at present far from having a general proof at our disposal. Section 3 therefore sets up a precise motivic setting in which semi-primality is guaranteed (Theorem 3.10).

Section 4 then leads up to Theorem 4.4.

One word about the Hodge-theoretic picture: an analogue of our main result Theorem 4.4 should certainly hold for the (generic) Hodge-theoretic realization $R_{\mathbf{H}}$. Unfortunately, this realization is at present not fully available. Note, however, that [Ivo16] provides a Hodge-theoretic realization for schemes which are smooth over $\mathbb{C}$. For the applications we have in mind [Wil17b], it will, however, be necessary to consider singular schemes. Similarly, we need the compatibility of $R_{\mathbf{H}}$ with the functors $f^{*}, f_{*}, f_{!}, f^{!}$. In the present work, we thus replace $R_{\mathbf{H}}$ by the (generic) Betti realization, the price to be paid being that we a priori lose the intrinsic notion of weights on the target of the realization. Nonetheless, we have sufficient control over the situation to prove the analogue of Theorem 4.3 as $R_{\ell}$, the restriction of the (generic) Betti realization to the category of motives of Abelian type, is conservative.

Conventions. Throughout the article, $F$ denotes a finite direct product of fields of characteristic zero. We fix a base scheme $\mathbb{B}$, which is of finite type over some excellent scheme of dimension at most two. By definition, schemes are $\mathbb{B}$-schemes which are separated and of finite type (in particular, they are excellent and Noetherian of finite dimension), morphisms between schemes are separated morphisms of $\mathbb{B}$-schemes, and a scheme is nilregular if the underlying reduced scheme is regular in the usual sense.

We use the triangulated, $\mathbb{Q}$-linear categories $\mathrm{DM}_{\mathrm{b}, c}(X)$ of constructible Beilinson motives over $X$ [CD12, Definition 15.1.1], indexed by schemes $X$ (always in the sense of the above conventions). In order to have an $F$-linear theory at one's disposal, one redoes the construction, but using $F$ instead of $\mathbb{Q}$ as coefficients [CD12, Section 15.2.5]. This yields triangulated, $F$ linear categories $\mathrm{DM}_{\mathrm{b}, c}(X)_{F}$ satisfying the $F$-linear analogues of the properties of $\mathrm{DM}_{\mathrm{b}, c}(X)$. In particular, these categories are pseudo-Abelian (see [Héb11, Section 2.10]). Furthermore, the canonical functor $\mathrm{DM}_{\mathrm{b}, c}(X) \otimes_{\mathbb{Q}} F \rightarrow \mathrm{DM}_{\mathrm{b}, c}(X)_{F}$ is fully faithful [CD12, Section 14.2.20]. As in [CD12], the symbol $\mathbb{1}_{X}$ is used to denote the unit for the tensor product in $\operatorname{DM}_{\mathrm{b}, c}(X)_{F}$. We shall employ the full formalism of six operations developed in op. cit. The reader may choose to consult [Héb11, Section 2] or [Wil12, Section 1] for concise presentations of this formalism.

\section{Conservativity and weight conservativity}

We make free use of the terminology of and basic results on weight structures [Bon11, Section 1.3].

Definition 2.1. Let $r: \mathcal{C}_{1} \rightarrow \mathcal{C}_{2}$ be an $F$-linear exact functor between $F$-linear triangulated categories equipped with weight structures $\left(\mathcal{C}_{1, w \leqslant 0}, \mathcal{C}_{1, w \geqslant 0}\right)$ and $\left(\mathcal{C}_{2, w \leqslant 0}, \mathcal{C}_{2, w \geqslant 0}\right)$. 


\section{J. Wildeshaus}

(a) The functor $r$ is said to be weight exact if

$$
r\left(\mathcal{C}_{1, w \leqslant 0}\right) \subset \mathcal{C}_{2, w \leqslant 0} \text { and } r\left(\mathcal{C}_{1, w \geqslant 0}\right) \subset \mathcal{C}_{2, w \geqslant 0}
$$

(b) If $r$ is weight exact, then we denote by $r_{w=0}$ the functor

$$
\mathcal{C}_{1, w=0} \longrightarrow \mathcal{C}_{2, w=0}
$$

induced by the restriction of $r$ to the heart $\mathcal{C}_{1, w=0}$.

Lemma 2.2. Let $r: \mathcal{C}_{1} \rightarrow \mathcal{C}_{2}$ be an $F$-linear exact functor between $F$-linear triangulated categories equipped with weight structures. We assume the following:

(1) The functor $r$ is weight exact.

(2) The functor $r_{w=0}: \mathcal{C}_{1, w=0} \rightarrow \mathcal{C}_{2, w=0}$ is full.

Then for any integer $n$ and any two objects $X \in \mathcal{C}_{1, w \leqslant n}$ and $Z \in \mathcal{C}_{1, w \geqslant n}$, the map

$$
r: \operatorname{Hom}_{\mathcal{C}_{1}}(X, Z) \longrightarrow \operatorname{Hom}_{\mathcal{C}_{2}}(r(X), r(Z))
$$

is surjective.

Proof. Fix weight filtrations

$$
X_{\leqslant n-1} \longrightarrow X \longrightarrow X_{n} \longrightarrow X_{\leqslant n-1}[1]
$$

and

$$
Z_{n} \longrightarrow Z \longrightarrow Z_{\geqslant n+1} \longrightarrow Z_{n}[1]
$$

of $X$ and $Z$, respectively, with $X_{n}, Z_{n} \in \mathcal{C}_{1, w=n}, X_{\leqslant n-1} \in \mathcal{C}_{1, w \leqslant n-1}$, and $Z_{\geqslant n+1} \in \mathcal{C}_{1, w \geqslant n+1}$. Given assumption (1), their images under $r$ are weight filtrations of $r(X)$ and $r(Z)$ of the same type.

Let $\beta: r(X) \rightarrow r(Z)$ be a morphism. By the orthogonality for the weight structure on $\mathcal{C}_{2}$, the morphism $\beta$ factors through a morphism $\beta^{\prime}: r\left(X_{n}\right) \rightarrow r\left(Z_{n}\right)$. The shift by $[-n]$ of the latter gives $\beta^{\prime}[-n]: r\left(X_{n}\right)[-n] \rightarrow r\left(Z_{n}\right)[-n]$, a morphism in $\mathcal{C}_{2, w=0}$. Given assumption (2), the morphism $\beta^{\prime}[-n]$ lies in the image of $r$. But then so do $\beta^{\prime}$ and $\beta$.

Before stating our first abstract result on conservativity, let us recall an important notion.

Definition 2.3. Let $\mathcal{C}$ be an $F$-linear triangulated category equipped with a weight structure $w$. Let $M \in \mathcal{C}$ and $n \in \mathbb{Z}$. A minimal weight filtration of $M$ concentrated at $n$ is a weight filtration

$$
M_{\leqslant n-1} \longrightarrow M \longrightarrow M_{\geqslant n} \stackrel{\delta}{\longrightarrow} M_{\leqslant n-1}[1]
$$

$\left(M_{\leqslant n-1} \in \mathcal{C}_{w \leqslant n-1}, M_{\geqslant n} \in \mathcal{C}_{w \geqslant n}\right)$ such that the morphism $\delta$ belongs to the radical [AK02, Définition 1.4.1] of $\mathcal{C}$; that is, $\delta \in \operatorname{rad}_{\mathcal{C}}\left(M_{\geqslant n}, M_{\leqslant n-1}[1]\right)$,

Any two minimal weight filtrations of the same object $M$ are related by an isomorphism (which in general is not unique) [Wil17a, proof of Theorem 2.2(b)].

Proposition 2.4. Let $r: \mathcal{C}_{1} \rightarrow \mathcal{C}_{2}$ be an $F$-linear exact functor between $F$-linear triangulated categories equipped with weight structures. We assume the following:

(1) The functor $r$ is weight exact.

(2) The functor $r_{w=0}: \mathcal{C}_{1, w=0} \rightarrow \mathcal{C}_{2, w=0}$ is full.

Then $r$ maps minimal weight filtrations to minimal weight filtrations. 


\section{WEIGHTS AND CONSERVATIVITY}

Proof. Let

$$
M_{\leqslant n-1} \longrightarrow M \longrightarrow M_{\geqslant n} \stackrel{\delta}{\longrightarrow} M_{\leqslant n-1}[1]
$$

be a minimal weight filtration of $M \in \mathcal{C}_{1}$ concentrated at $n$. Thanks to assumption (1), the exact triangle $r(*)$ is a weight filtration of $r(M)$.

According to Lemma 2.2, any morphism $r\left(M_{\leqslant n-1}[1]\right) \rightarrow r\left(M_{\geqslant n}\right)$ lies in the image of $r$. Using this information and applying the definition, one sees that $r(\delta)$ belongs to the radical of $\mathcal{C}_{2}$ since $\delta$ belongs to the radical of $\mathcal{C}_{1}$. Therefore, $r(*)$ is a minimal weight filtration of $r(M)$ concentrated at $n$.

TheOREM 2.5. Let $r: \mathcal{C}_{1} \rightarrow \mathcal{C}_{2}$ be an $F$-linear exact functor between $F$-linear triangulated categories equipped with weight structures. We assume the following:

(1) The weight structure on $\mathcal{C}_{1}$ is bounded; that is, its heart $\mathcal{C}_{1, w=0}$ generates $\mathcal{C}_{1}$ as a triangulated category.

(2) The heart $\mathcal{C}_{1, w=0}$ is semi-primary [AK02, Définition 2.3.1] and pseudo-Abelian.

(3) The functor $r$ is weight exact.

(4) The functor $r_{w=0}$ is full.

(5) The functor $r_{w=0}$ is conservative.

Then $r$ is conservative.

Proof. The functor $r$ being an exact functor between triangulated categories, it suffices to show that only the zero object is mapped to zero under $r$. Thus, let $M \in \mathcal{C}_{1}$, and assume $r(M)=0$.

Given assumption (1), the category $\mathcal{C}_{1}$ is pseudo-Abelian if and only if its heart $\mathcal{C}_{1, w=0}$ is [Bon10, Lemma 5.2.1]. Given assumption (2), we are thus in the abstract situation studied in [Wil17a, Section 2], meaning in particular that minimal weight filtrations do exist for any object of $\mathcal{C}_{1}$ [Wil17a, Theorem 2.2(a)]. Let

$$
M_{\leqslant-1} \longrightarrow M \longrightarrow M_{\geqslant 0} \stackrel{\delta}{\longrightarrow} M_{\leqslant-1}[1]
$$

be a minimal weight filtration of $M$; that is, $M_{\leqslant-1} \in \mathcal{C}_{1, w \leqslant-1}, M_{\geqslant 0} \in \mathcal{C}_{1, w \geqslant 0}$, and the morphism $\delta$ belongs to the radical of $\mathcal{C}_{1}$.

On the one hand, the triangle $r(*)$ is exact, and $r(M)=0$. Therefore, the morphism $r(\delta)$ is an isomorphism. On the other hand, according to Proposition 2.4 (applicable thanks to hypotheses (3) and (4)), $r(\delta)$ belongs to the radical of $\mathcal{C}_{2}$. But then $r(\delta)$ must be the zero morphism $0 \rightarrow 0$, meaning that both $M_{\leqslant-1}$ and $M_{\geqslant 0}$ are mapped to zero under $r$.

According to hypothesis (1), the weight structure on $\mathcal{C}_{1}$ is bounded. Therefore, the above procedure, successively applied to minimal weight filtrations concentrated at integers different from zero, allows us to reduce to the case where $M$ is pure of some weight, say $n$. But then $M[-n]$ belongs to $\mathcal{C}_{1, w=0}$. Now apply assumption (5).

Remark 2.6. The analogue of Theorem 2.5 for triangulated categories equipped with $t$-structures holds and requires only the analogues of assumptions (1), (3), and (5).

Recall the following.

Definition 2.7 ([Wil09, Definitions 1.6 and 1.10]). Let $\mathcal{C}$ be an $F$-linear triangulated category equipped with a weight structure $w$. Let $M \in \mathcal{C}$, and let $\alpha \leqslant \beta$ be two integers (which may be identical). 


\section{J. WILDESHAUS}

(a) A weight filtration of $M$ avoiding weights $\alpha, \alpha+1, \ldots, \beta$ is an exact triangle

$$
M_{\leqslant \alpha-1} \longrightarrow M \longrightarrow M_{\geqslant \beta+1} \longrightarrow M_{\leqslant \alpha-1}[1]
$$

in $\mathcal{C}$ with $M_{\leqslant \alpha-1} \in \mathcal{C}_{w \leqslant \alpha-1}$ and $M_{\geqslant \beta+1} \in \mathcal{C}_{w \geqslant \beta+1}$.

(b) We say that $M \in \mathcal{C}$ does not have weights $\alpha, \alpha+1, \ldots, \beta$, or that $M$ is without weights $\alpha, \alpha+1, \ldots, \beta$, if it admits a weight filtration avoiding weights $\alpha, \alpha+1, \ldots, \beta$.

We leave it to the reader to verify that any weight filtration avoiding weights $\alpha, \alpha+1, \ldots, \beta$ is a minimal weight filtration concentrated at $n$, for any integer $n$ between $\alpha$ and $\beta+1$.

The following result sharpens Theorem 2.5; it will, however, not be needed in the remainder of this paper.

TheOREM 2.8. Let $r: \mathcal{C}_{1} \rightarrow \mathcal{C}_{2}$ be an $F$-linear exact functor between $F$-linear triangulated categories equipped with weight structures. The assumptions (1)-(5) are the same as in Theorem 2.5. Then $r$ is weight conservative; that is, it detects weights. More precisely, let $M \in \mathcal{C}_{1}$, and let $\alpha \leqslant \beta$ be two integers.

(a) The object $M$ lies in the heart $\mathcal{C}_{1, w=0}$ if and only if $r(M)$ lies in the heart $\mathcal{C}_{2, w=0}$.

(b) The object $M$ lies in $\mathcal{C}_{1, w \leqslant \alpha}$ if and only if $r(M)$ lies in $\mathcal{C}_{2, w \leqslant \alpha}$.

(c) The object $M$ lies in $\mathcal{C}_{1, w \geqslant \beta}$ if and only if $r(M)$ lies in $\mathcal{C}_{2, w \geqslant \beta}$.

(d) The object $M$ is without weights $\alpha, \alpha+1, \ldots, \beta$ if and only if $r(M)$ is without weights $\alpha, \alpha+1, \ldots, \beta$.

Proof. According to assumption (1), the weight structure on $\mathcal{C}_{1}$ is bounded, and the "only if" parts of statements (a)-(d) are true. It therefore suffices to prove the "if" part of statement (d).

Consider minimal weight filtrations concentrated at weight $\alpha$ and at weight $\beta+1$, respectively:

$$
\begin{gathered}
M_{\leqslant \alpha-1} \longrightarrow M \longrightarrow M_{\geqslant \alpha} \stackrel{\delta_{\alpha}}{\longrightarrow} M_{\leqslant \alpha-1}[1], \\
M_{\leqslant \beta} \longrightarrow M \longrightarrow M_{\geqslant \beta+1} \stackrel{\delta_{\beta+1}}{\longrightarrow} M_{\leqslant \beta}[1] .
\end{gathered}
$$

By orthogonality, the identity on $M$ extends to a morphism of exact triangles

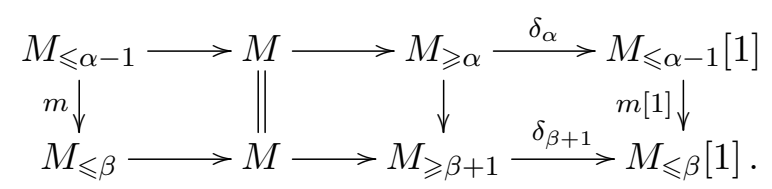

By Proposition 2.4, the image under $r$ of the above is a morphism relating minimal weight filtrations concentrated at weight $\alpha$ and at weight $\beta+1$, respectively:

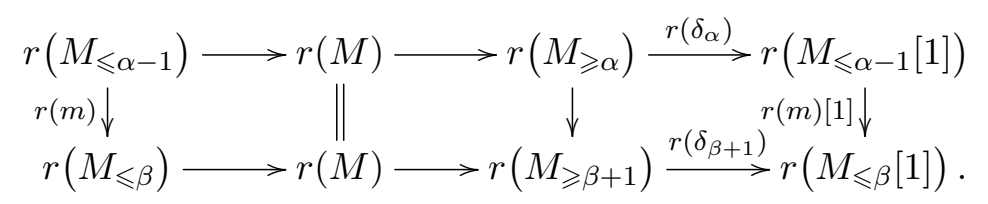

Thus, both $r\left(\delta_{\alpha}\right)$ and $r\left(\delta_{\beta+1}\right)$ lie in the radical of $\mathcal{C}_{2}$.

But $r(M)$ is supposed to be without weights $\alpha, \alpha+1, \ldots, \beta$, meaning that there is a minimal weight filtration of $r(M)$ which is concentrated at any integer between $\alpha$ and $\beta+1$. The unicity of minimal weight filtrations in $\mathcal{C}_{2}$ [Wil17a, proof of Theorem 2.2(b)] shows that this latter weight filtration is isomorphic to both the top and the bottom of the above diagram, meaning in 


\section{WEIGHTS AND CONSERVATIVITY}

particular that they are (abstractly) isomorphic to each other. In particular, the object $r\left(M_{\leqslant \beta}\right)$ belongs to $\mathcal{C}_{2, w \leqslant \alpha-1}$. Orthogonality then allows us to extend the identity on $r(M)$ to a morphism of exact triangles

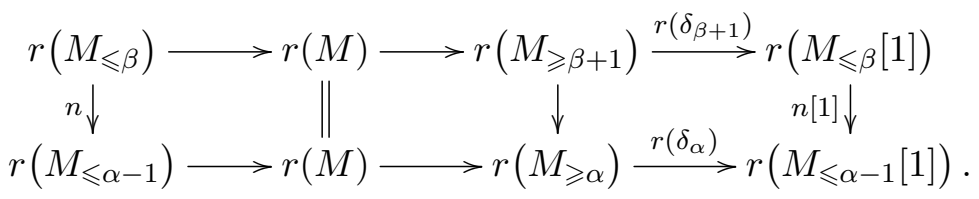

Using the fact that both $r\left(\delta_{\alpha}\right)$ and $r\left(\delta_{\beta+1}\right)$ lie in the radical, one shows that both compositions $n \circ r(m)$ and $r(m) \circ n$ are automorphisms; in particular, $r(m)$ is an isomorphism.

But then (Theorem 2.5), so is $m$ itself. This yields a weight filtration

$$
M_{\leqslant \alpha-1} \longrightarrow M \longrightarrow M_{\geqslant \beta+1} \longrightarrow M_{\leqslant \alpha-1}[1]
$$

of $M$ avoiding weights $\alpha, \alpha+1, \ldots, \beta$.

The second half of the present section treats conservativity in a slightly different context. The source of the functors in question remains equipped with a weight structure. But their target is only supposed to be Abelian. Recall [Bon10, Proposition 2.1.2(1)] that any (covariant) additive functor $\mathcal{H}$ from a triangulated category $\mathcal{C}$ carrying a weight structure $w$ to an Abelian category $\mathfrak{A}$ admits a canonical weight filtration by sub-functors

$$
\cdots \subset W_{n} \mathcal{H} \subset W_{n+1} \mathcal{H} \subset \cdots \subset \mathcal{H} .
$$

According to [Bon10, Definition 2.1.1] (use the normalization of [Bon11, Definition 1.3.1] for the signs of the weights), for an object $M$ of $\mathcal{C}$ and $n \in \mathbb{Z}$, the sub-object $W_{n} \mathcal{H}(M) \subset \mathcal{H}(M)$ is defined as the image of the morphism $\mathcal{H}\left(\iota_{w \leqslant n}\right)$, for any weight filtration

$$
M_{w \leqslant n} \stackrel{\iota_{w \leqslant n}}{\longrightarrow} M \longrightarrow M_{w \geqslant n+1} \longrightarrow M_{w \leqslant n}[1]
$$

(with $M_{w \leqslant n} \in \mathcal{C}_{w \leqslant n}$ and $M_{w \geqslant n+1} \in \mathcal{C}_{w \geqslant n+1}$ ). For any $m \in \mathbb{Z}$, one defines

$$
\mathcal{H}^{m}: \mathcal{C} \longrightarrow \mathfrak{A}, \quad M \longrightarrow \mathcal{H}(X[m]) ;
$$

according to the usual convention, the weight filtration of $\mathcal{H}^{m}(M)$ equals the weight filtration of $\mathcal{H}(X[m])$; that is, it differs by décalage from the intrinsic weight filtration of the covariant additive functor $\mathcal{H}^{m}$.

The proof of the following result is formal, and therefore left to the reader (cf. [Wil15, Lemma 1.11]).

Lemma 2.9. Let $\mathcal{H}: \mathcal{C}_{1} \rightarrow \mathcal{C}_{3}$ be an $F$-linear functor from an $F$-linear triangulated category $\mathcal{C}_{1}$ equipped with a weight structure to an $F$-linear Abelian category $\mathcal{C}_{3}$. We assume the following:

(1) Any morphism in the image of $\mathcal{H}$ is strict with respect to the weight filtration of $\mathcal{H}$.

(2) The restriction of $\mathcal{H}$ to the heart $\mathcal{C}_{1, w=0}$ maps the radical to zero.

Then for any integer $n$ and any two objects $X \in \mathcal{C}_{1, w \leqslant n}$ and $Z \in \mathcal{C}_{1, w \geqslant n}$, the map

$$
\mathcal{H}: \operatorname{Hom}_{\mathcal{C}_{1}}(Z, X) \longrightarrow \operatorname{Hom}_{\mathcal{C}_{3}}(\mathcal{H}(Z), \mathcal{H}(X))
$$

maps the radical to zero.

Theorem 2.10. Let $\mathcal{H}: \mathcal{C}_{1} \rightarrow \mathcal{C}_{3}$ be an $F$-linear homological functor from an $F$-linear triangulated category $\mathcal{C}_{1}$ equipped with a weight structure to an $F$-linear Abelian category $\mathcal{C}_{3}$. We assume the following: 


\section{J. Wildeshaus}

(1) The weight structure on $\mathcal{C}_{1}$ is bounded.

(2) The heart $\mathcal{C}_{1, w=0}$ is semi-primary and pseudo-Abelian.

(3) Any morphism in the image of $\mathcal{H}$ is strict with respect to the weight filtration of $\mathcal{H}$.

(4) The restriction of $\mathcal{H}$ to the heart $\mathcal{C}_{1, w=0}$ maps the radical to zero.

(5) Zero is the only object of the heart $\mathcal{C}_{1, w=0}$ mapped to zero under (the restriction to $\mathcal{C}_{1, w=0}$ of) the functor $\left(\mathcal{H}^{m}\right)_{m \in \mathbb{Z}}$.

Then $\left(\mathcal{H}^{m}\right)_{m \in \mathbb{Z}}$ is conservative.

Proof. Let $M \in \mathcal{C}_{1}$. Let

$$
M_{\leqslant-1} \longrightarrow M \longrightarrow M_{\geqslant 0} \stackrel{\delta}{\longrightarrow} M_{\leqslant-1}[1]
$$

be a minimal weight filtration of $M$ (here, we use assumptions (1) and (2)). According to Lemma 2.9 (applicable thanks to hypotheses (3) and (4)), we have $\mathcal{H}(\delta)=0$. The functor $\mathcal{H}$ is supposed to be homological; hence, if $\mathcal{H}(M)$ is zero, then both $\mathcal{H}\left(M_{\leqslant-1}\right)$ and $\mathcal{H}\left(M_{\geqslant 0}\right)$ are zero. Similarly, if $\mathcal{H}^{m}(M)$ is zero for some $m \in \mathbb{Z}$, then so are $\mathcal{H}^{m}\left(M_{\leqslant-1}\right)$ and $\mathcal{H}^{m}\left(M_{\geqslant 0}\right)$.

Now, let $M \in \mathcal{C}_{1}$, and assume $\mathcal{H}^{m}(M)=0$ for all $m \in \mathbb{Z}$. We need to show that $M=0$. According to hypothesis (1), the weight structure on $\mathcal{C}_{1}$ is bounded. Therefore, the above procedure, successively applied to minimal weight filtrations concentrated at integers different from zero, allows us to reduce to the case where $M$ is pure of some weight, say $n$. But then $M[-n]$ belongs to $\mathcal{C}_{1, w=0}$. Now, apply assumption (5).

As before, there is a version "with weights" of the above.

TheOREM 2.11. Let $\mathcal{H}: \mathcal{C}_{1} \rightarrow \mathcal{C}_{3}$ be an $F$-linear homological functor from an $F$-linear triangulated category $\mathcal{C}_{1}$ equipped with a weight structure to an $F$-linear Abelian category $\mathcal{C}_{3}$. The assumptions (1)-(5) are the same as in Theorem 2.10. Then $\left(\mathcal{H}^{m}\right)_{m \in \mathbb{Z}}$ is weight conservative; that is, it detects weights. More precisely, let $M \in \mathcal{C}_{1}$, and let $\alpha \leqslant \beta$ be two integers.

(a) The object $M$ lies in the heart $\mathcal{C}_{1, w=0}$ if and only if $\mathcal{H}^{n}(M)$ is pure of weight $n$ for all $n \in \mathbb{Z}$.

(b) The object $M$ lies in $\mathcal{C}_{1, w \leqslant \alpha}$ if and only if $\mathcal{H}^{n}(M)$ is of weights at most $n+\alpha$ for all $n \in \mathbb{Z}$.

(c) The object $M$ lies in $\mathcal{C}_{1, w \geqslant \beta}$ if and only if $\mathcal{H}^{n}(M)$ is of weights at least $n+\beta$ for all $n \in \mathbb{Z}$.

(d) The object $M$ is without weights $\alpha, \alpha+1, \ldots, \beta$ if and only if $\mathcal{H}^{n}(M)$ is without weights $n+\alpha, n+\alpha+1, \ldots, n+\beta$ for all $n \in \mathbb{Z}$.

Proof. According to assumption (1), the weight structure on $\mathcal{C}_{1}$ is bounded, and the "only if" parts of statements (a)-(d) are true. It therefore suffices to prove the "if" part of statement (d).

Consider minimal weight filtrations concentrated at weight $\alpha$ and at weight $\beta+1$, respectively:

$$
\begin{gathered}
M_{\leqslant \alpha-1} \longrightarrow M \longrightarrow M_{\geqslant \alpha} \stackrel{\delta_{\alpha}}{\longrightarrow} M_{\leqslant \alpha-1}[1], \\
M_{\leqslant \beta} \longrightarrow M \longrightarrow M_{\geqslant \beta+1} \stackrel{\delta_{\beta+1}}{\longrightarrow} M_{\leqslant \beta}[1] .
\end{gathered}
$$

By orthogonality, the identity on $M$ extends to a morphism of exact triangles

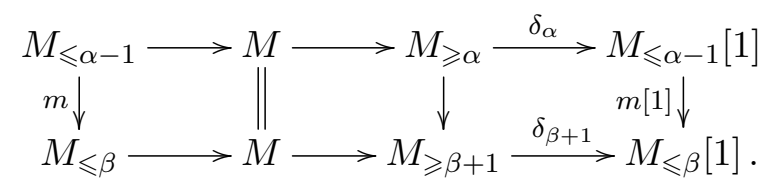




\section{WEIGHTS AND CONSERVATIVITY}

By Lemma 2.9 , both $\mathcal{H}^{*}\left(\delta_{\alpha}\right)$ and $\mathcal{H}^{*}\left(\delta_{\beta+1}\right)$ are zero. Thus, the above morphism of exact triangles induces a morphism of exact sequences

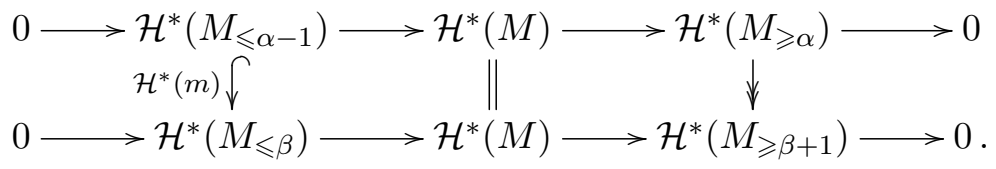

Our hypothesis on weights avoided in $\mathcal{H}^{*}(M)$ implies that the monomorphism $\mathcal{H}^{*}(m)$ is in fact an isomorphism.

But then (Theorem 2.10), so is $m$ itself. This yields a weight filtration

$$
M_{\leqslant \alpha-1} \longrightarrow M \longrightarrow M_{\geqslant \beta+1} \longrightarrow M_{\leqslant \alpha-1}[1]
$$

of $M$ avoiding weights $\alpha, \alpha+1, \ldots, \beta$.

\section{Relative motives of Abelian type}

Let $S$ be a scheme (in the sense of the conventions fixed in our introduction).

Definition 3.1. A good stratification of $S$ indexed by a finite set $\mathfrak{S}$ is a collection of locally closed sub-schemes $S_{\sigma}$ indexed by $\sigma \in \mathfrak{S}$ such that

$$
S=\coprod_{\sigma \in \mathfrak{S}} S_{\sigma}
$$

on the set-theoretic level, and such that the closure $\overline{S_{\sigma}}$ of any stratum $S_{\sigma}$ is a union of strata $S_{\tau}$.

In the setting of Definition 3.1, we shall often write $S(\mathfrak{S})$ instead of $S$. Recall the following result.

Theorem 3.2 ([Wil17a, Theorem 4.5(a)]). Let $S(\mathfrak{S})=\coprod_{\sigma \in \mathfrak{S}} S_{\sigma}$ be a good stratification of a scheme $S(\mathfrak{S})$. Assume the following for all $\sigma \in \mathfrak{S}:(\alpha)$ the stratum $S_{\sigma}$ is nilregular; $(\beta)$ for any stratum $i_{\tau}: S_{\tau} \hookrightarrow \overline{S_{\sigma}}$ contained in the closure $\overline{S_{\sigma}}$ of $S_{\sigma}$, the functor

$$
i_{\tau}^{!}: \mathrm{DM}_{\mathrm{E}, c}\left(\overline{S_{\sigma}}\right)_{F} \longrightarrow \mathrm{DM}_{\mathrm{E}, c}\left(S_{\tau}\right)_{F}
$$

maps $\mathbb{1}_{\overline{S_{\sigma}}}$ to a Tate motive over $S_{\tau}$. Then the categories $\operatorname{DMT}\left(S_{\sigma}\right)_{F}$ of Tate motives over $S_{\sigma}$ [Lev10, Section 3.3], for $\sigma \in \mathfrak{S}$, can be glued to give a full, triangulated sub-category $\operatorname{DMT}_{\mathfrak{S}}(S(\mathfrak{S}))_{F}$ of $\operatorname{DM}_{\mathrm{E}, c}(S(\mathfrak{S}))_{F}$.

Recall [Wil17a, Remark 4.7] that thanks to absolute purity [CD12, Theorem 14.4.1], hypotheses $(\alpha)$ and $(\beta)$ from Theorem 3.2 are satisfied as soon as the closures $\overline{S_{\sigma}}$ of all strata $S_{\sigma}$, for $\sigma \in \mathfrak{S}$, are nilregular.

Definition 3.3. Let $S(\mathfrak{S})=\coprod_{\sigma \in \mathfrak{S}} S_{\sigma}$ be a good stratification of a scheme $S(\mathfrak{S})$. Assume the following for all $\sigma \in \mathfrak{S}:(\alpha) S_{\sigma}$ is nilregular; $(\beta)$ for any $i_{\tau}: S_{\tau} \hookrightarrow \overline{S_{\sigma}}$, the functor $i_{\tau}^{!}$maps $\mathbb{1}_{\overline{S_{\sigma}}}$ to a Tate motive over $S_{\tau}$. The category $\operatorname{DMT}_{\mathfrak{S}}(S(\mathfrak{S}))_{F}$ of Theorem 3.2 is called the category of $\mathfrak{S}$-constructible Tate motives over $S(\mathfrak{S})$.

According to [Wil17a, Theorem 4.5(d)], the category $\operatorname{DMT}_{\mathfrak{S}}(S(\mathfrak{S}))_{F}$ is pseudo-Abelian. Now, let $S(\mathfrak{S})=\coprod_{\sigma \in \mathfrak{S}} S_{\sigma}$ and $Y(\Phi)=\coprod_{\varphi \in \Phi} Y_{\varphi}$ be good stratifications of schemes $S(\mathfrak{S})$ and $Y(\Phi)$, respectively. 


\section{J. Wildeshaus}

Definition 3.4. A morphism $\pi: S(\mathfrak{S}) \rightarrow Y(\Phi)$ is said to be a morphism of good stratifications if the pre-image $\pi^{-1}\left(Y_{\varphi}\right)$ of any stratum $Y_{\varphi}$ of $Y(\Phi)$, with $\varphi \in \Phi$, is a union of strata $S_{\sigma}$.

Definition 3.5. (a) A morphism $\pi: S(\mathfrak{S}) \rightarrow Y(\Phi)$ of good stratifications is said to be of Abelian type if it is proper and if the following conditions are satisfied:

(1) All strata $Y_{\varphi}$ and $S_{\sigma}$, for $\varphi \in \Phi$ and $\sigma \in \mathfrak{S}$, are nilregular, and for any $i_{\tau}: S_{\tau} \hookrightarrow \overline{S_{\sigma}}$, the functor $i_{\tau}^{!}$maps $\mathbb{1}_{\overline{S_{\sigma}}}$ to a Tate motive over $S_{\tau}$.

(2) For all $\sigma \in \mathfrak{S}$ such that $S_{\sigma}$ is a stratum of $\pi^{-1}\left(Y_{\varphi}\right)$, the morphism $\pi_{\sigma}: S_{\sigma} \rightarrow Y_{\varphi}$ can be factored as

$$
\pi_{\sigma}=\pi_{\sigma}^{\prime} \circ \pi_{\sigma}^{\prime \prime}: S_{\sigma} \stackrel{\pi_{\sigma}^{\prime \prime}}{\longrightarrow} B_{\sigma} \stackrel{\pi_{\sigma}^{\prime}}{\longrightarrow} Y_{\varphi}
$$

so that the motive

$$
\pi_{\sigma, *}^{\prime \prime} \mathbb{1}_{S_{\sigma}} \in \mathrm{DM}_{\mathrm{L}, c}\left(B_{\sigma}\right)_{F}
$$

belongs to the category $\operatorname{DMT}\left(B_{\sigma}\right)_{F}$ of Tate motives over $B_{\sigma}$, the morphism $\pi_{\sigma}^{\prime}$ is proper and smooth, and its pull-back to any geometric point of $Y_{\varphi}$ lying over a generic point is isomorphic to a finite disjoint union of Abelian varieties.

(b) Let $Y$ be a scheme equipped with a good stratification $\Phi$ with nilregular strata. Define the category $\mathrm{DM}_{\mathrm{E}, c, \Phi}^{A b}(Y)_{F}$ as the strict, full, dense, $F$-linear triangulated sub-category of $\mathrm{DM}_{\mathrm{E}, c}(Y)_{F}$ generated by the images under $\pi_{*}$ of the objects of $\operatorname{DMT}_{\mathfrak{S}}(S(\mathfrak{S}))_{F}$, where $\pi: S(\mathfrak{S}) \rightarrow Y(\Phi)$ runs through the morphisms of Abelian type with target equal to $Y=Y(\Phi)$. Objects of $\mathrm{DM}_{\mathrm{E}, c, \Phi}^{A b}(Y)_{F}$ are called $\Phi$-constructible motives of Abelian type over $Y$.

(c) Let $Y$ be a nilregular scheme. Set

$$
\mathrm{DM}_{\mathrm{E}, c}^{A b}(Y)_{F}:=\mathrm{DM}_{\mathrm{E}, c, \Phi}^{A b}(Y)_{F}
$$

for the trivial stratification $\Phi=\{\varphi\}$.

Since the category $\mathrm{DM}_{\mathrm{E}, c}(Y)_{F}$ is pseudo-Abelian (see [Héb11, Section 2.10]), so is $\mathrm{DM}_{\mathrm{E}, c, \Phi}^{A b}(Y)_{F}$.

If a nilregular scheme $Y$ is equipped with a good stratification $\Phi$ with nilregular strata, then by [Wil17a, Theorem 4.5(b)] and [CD12, Theorem 15.2.4], the category $\operatorname{DM}_{\mathrm{E}, c, \Phi}^{A b}(Y)_{F}$ is closed under duality.

Next, we need to discuss weight structures.

THEOREM 3.6. Let $Y$ be a scheme equipped with a good stratification $\Phi$ with nilregular strata.

(a) The motivic weight structure $w$ on $\mathrm{DM}_{\mathrm{b}, c}(Y(\Phi))_{F}$ ([Héb11, Théorème 3.3], [Bon14, Theorem 2.1.1]) induces a weight structure, still denoted by the same letter $w$, on $\operatorname{DM}_{\mathrm{E}, c, \Phi}^{A b}(Y)_{F}$.

(b) The motivic weight structure on $\mathrm{DM}_{\mathrm{b}, c, \Phi}^{A b}(Y)_{F}$ is bounded.

Proof. We imitate the proof of [Wil17a, Corollary 4.11]. Let $\mathcal{K}$ be the strict, full, $F$-linear subcategory of $\mathrm{DM}_{\mathrm{L}, c}(Y(\Phi))_{F}$ of finite direct sums of motives isomorphic to images under $\pi_{*}$ of objects in

$$
\operatorname{CHMT}_{\mathfrak{S}}(S(\mathfrak{S}))_{F}:=\operatorname{DMT}_{\mathfrak{S}}(S(\mathfrak{S}))_{F} \cap C H M(S(\mathfrak{S}))_{F},
$$

for morphisms $\pi: S(\mathfrak{S}) \rightarrow Y(\Phi)$ of Abelian type. Denote by $\mathcal{D}$ the triangulated category generated by $\mathcal{K}$.

According to our definition and [Wil17a, Corollary 4.12(b)], the category $\mathcal{D}$ equals the strict, full, $F$-linear triangulated sub-category of $\mathrm{DM}_{\mathrm{L}, c}(Y)_{F}$ generated by the images under $\pi_{*}$ of the objects of $\operatorname{DMT}_{\mathfrak{S}}(S(\mathfrak{S}))_{F}$, where $\pi: S(\mathfrak{S}) \rightarrow Y(\Phi)$ runs through the morphisms of Abelian type. 


\section{WEIGHTS AND CONSERVATIVITY}

Following [Wil17a, Remark 4.4], the motivic weight structure induces a bounded weight structure on $\mathcal{D}$ whose heart contains $\mathcal{K}$.

Repeat the same argument with the pseudo-Abelian completion $\mathcal{K}^{\natural}$ instead of $\mathcal{K}$. We get a bounded weight structure, induced by the motivic weight structure, on a triangulated subcategory $\mathcal{D}^{\natural}$ of $\operatorname{DM}_{\mathrm{E}, c}(Y(\Phi))_{F}$.

Our claim is implied by [Bon10, Proposition 5.2.2], which states that $\mathcal{D}^{\natural}$ is the pseudo-Abelian completion of $\mathcal{D}$, hence equal to $\operatorname{DM}_{\mathrm{E}, c, \Phi}^{A b}(Y)_{F}$.

Definition 3.7. (a) Let $Y$ be a scheme equipped with a good stratification $\Phi$ with nilregular strata. A $\Phi$-constructible Chow motive of Abelian type over $Y$ is an object of

$$
\mathrm{CHM}_{\Phi}^{A b}(Y)_{F}:=D M_{\mathrm{E}, c, \Phi}^{A b}(Y)_{F, w=0} .
$$

(b) Let $Y$ be a nilregular scheme. Set

$$
\mathrm{CHM}^{A b}(Y)_{F}:=\operatorname{CHM}_{\Phi}^{A b}(Y)_{F}
$$

for the trivial stratification $\Phi=\{\varphi\}$.

Note that since $\operatorname{DM}_{\mathrm{E}, c, \Phi}^{A b}(Y)_{F}$ is pseudo-Abelian, so is $\operatorname{CHM}_{\Phi}^{A b}(Y)_{F}$. Using [Bon10, Theorem 4.3.2(II)], let us extract the following from the proof of Theorem 3.6.

LEMma 3.8. Let $Y$ be a scheme equipped with a good stratification $\Phi$ with nilregular strata. Then the strict, full, pseudo-Abelian, F-linear sub-category $\mathrm{CHM}_{\Phi}^{A b}(Y)_{F}$ of $\mathrm{DM}_{\mathrm{\textrm {b }}, c}(Y)_{F}$ is generated by the images under $\pi_{*}$ of objects in $\operatorname{CHMT}_{\mathfrak{S}}(S(\mathfrak{S}))_{F}$, where $\pi: S(\mathfrak{S}) \rightarrow Y(\Phi)$ runs through the morphisms of Abelian type with target $Y(\Phi)$.

In order to have the main result from [Wil17a] at our disposal, let us check its hypotheses.

Proposition 3.9. Let $\pi: S(\mathfrak{S}) \rightarrow Y(\Phi)$ be a morphism of Abelian type.

(a) Let $\sigma \in \mathfrak{S}$ be such that $S_{\sigma}$ is a stratum of $\pi^{-1}\left(Y_{\varphi}\right)$, and let

$$
\pi_{\sigma}=\pi_{\sigma}^{\prime} \circ \pi_{\sigma}^{\prime \prime}: S_{\sigma} \stackrel{\pi_{\sigma}^{\prime \prime}}{\longrightarrow} B_{\sigma} \stackrel{\pi_{\sigma}^{\prime}}{\longrightarrow} Y_{\varphi}
$$

be a factorization of the morphism $\pi_{\sigma}: S_{\sigma} \rightarrow Y_{\varphi}$ as in Definition 3.5(a)(2). Then the smooth Chow motive over $Y_{\varphi}$ [Lev09, Definition 5.16]

$$
\pi_{\sigma, *}^{\prime} \mathbb{1}_{B_{\sigma}} \in \mathrm{CHM}^{s}\left(Y_{\varphi}\right)_{F}
$$

is finite-dimensional [AK02, Définition 9.1.1] (cf. [Kim05, Definition 3.7]).

(b) The morphism $\pi$ satisfies the assumptions of [Wil17a, Main Theorem 5.4].

Proof. The morphism $\pi_{\sigma}^{\prime}$ is proper and smooth, and its pull-back to any geometric point of $Y_{\varphi}$ lying over a generic point is isomorphic to a finite disjoint union of Abelian varieties. According to [O'Su11, end of Section 5.1, pp. 54-55], finite-dimensionality can be checked after base change to the geometric generic points of $Y_{\varphi}$. Now, apply [Kün94, Theorem 3.3.1]. This establishes part (a) of the claim.

But given Definition 3.5, part (a) is all that is needed in order to show that the assumptions of [Wil17a, Main Theorem 5.4] are satisfied.

We thus get the following structural result. 


\section{J. Wildeshaus}

TheOREM 3.10. Let $Y$ be a scheme equipped with a good stratification $Y=Y(\Phi)$ with nilregular strata. Then the $F$-category $\mathrm{CHM}_{\Phi}^{A b}(Y)_{F}$ of $\Phi$-constructible Chow motives of Abelian type over $Y$ is semi-primary (and pseudo-Abelian).

Proof. Given [AK02, Proposition 2.3.4(c)], our claim follows from Lemma 3.8, Proposition 3.9(b), and [Wil17a, Main Theorem 5.4].

Here is our first result on conservativity in the motivic context.

Theorem 3.11. Fix a generic point $\operatorname{Spec} k \hookrightarrow \mathbb{B}$ of the base scheme $\mathbb{B}$. Let $Y=Y(\Phi)=\coprod_{\varphi \in \Phi} Y_{\varphi}$ be a good stratification with nilregular strata such that the generic points of all $Y_{\varphi}$ lie over Spec $k \hookrightarrow \mathbb{B}$. Denote the base change of $Y$ to Spec $k$ by $Y_{k}$. Then the inverse image functor

$$
\mathrm{DM}_{\mathrm{B}, c, \Phi}^{A b}(Y)_{F} \longrightarrow \mathrm{DM}_{\mathrm{B}, c, \Phi}^{A b}\left(Y_{k}\right)_{F}
$$

is conservative.

Proof. According to [CD12, Theorem 14.3.3], the categories $\operatorname{DM}_{\mathrm{E}, c}(\bullet)_{F}$ are separated in the sense of [CD12, Definition 2.1.7]. Therefore, it suffices to check the claim after application of the inverse image functors to all $Y_{\varphi}$. Given proper base change [CD12, Theorem 2.4.50(4)], we are thus reduced to the case where the stratification $\Phi$ consists of a single stratum: $Y(\Phi)=Y_{\varphi}$. Recall that, by assumption, the scheme $Y_{\varphi}$ is nilregular.

According to Lemma 3.8 and [Will7a, Proposition 5.5], every object of $\operatorname{CHM}_{\Phi}^{A b}(Y)_{F}$ is a direct factor of a finite direct sum of objects isomorphic to $\pi_{*}^{\prime} \mathbb{1}_{B}(p)[2 p]$, for $p \in \mathbb{Z}$ and proper and smooth morphisms $\pi^{\prime}: B \rightarrow Y_{\varphi}$.

In particular, $\mathrm{CHM}_{\Phi}^{A b}(Y)_{F}$ is contained in the category of smooth Chow motives over $Y_{\varphi}$. By assumption, the morphism $Y_{\varphi, k} \rightarrow Y_{\varphi}$ is dominant. The conservativity of the restriction of the inverse image

$$
\alpha^{*}: \mathrm{DM}_{\mathrm{E}, c, \Phi}^{A b}(Y)_{F} \longrightarrow \mathrm{DM}_{\mathrm{b}, c, \Phi}^{A b}\left(Y_{k}\right)_{F}
$$

to $\mathrm{CHM}_{\Phi}^{A b}(Y)_{F}$ thus follows from [O'Su11, end of Section 5.1, pp. 54-55].

To treat the full triangulated category $\mathrm{DM}_{\mathrm{b}, c, \Phi}^{A b}(Y)_{F}$, note that according to Theorem 3.6(b), its weight structure is bounded. By Theorem 3.10, the heart of the weight structure is semiprimary and pseudo-Abelian. Furthermore, the functor $\alpha^{*}$ is weight exact. According to [O'Su11, Proposition 5.1.1], the restriction of the functor $\alpha^{*}$ to the heart $\operatorname{CHM}_{\Phi}^{A b}(Y)_{F}$ is full.

Thus, the assumptions of Theorem 2.5 are all satisfied.

\section{Realizations}

This section will be devoted to realizations. We fix a generic point $\mathbf{S p e c} k$ of our base scheme $\mathbb{B}$ and assume that we are in one of the following situations:

Case $1: k$ is embedded into $\mathbb{C}$ via a morphism $\eta: k \hookrightarrow \mathbb{C}$. The embedding yields a geometric point of $\mathbb{B}$, denoted by the same symbol

$$
\eta: \operatorname{Spec} \mathbb{C} \longrightarrow \operatorname{Spec} k \longleftrightarrow \mathbb{B} .
$$

The Betti realization is defined in [Ayo10, Définition 2.1]. It is a family of covariant exact functors

$$
R_{\eta, Z}: \mathbf{S H}(Z) \longrightarrow \mathrm{D}(Z)
$$




\section{WEIGHTS AND CONSERVATIVITY}

indexed by quasi-projective $k$-schemes $Z$. The source of $R_{\eta, Z}$ is the stable homotopy category of $Z$-schemes [Ayo07, Section 4.5]. Its target is the derived category of the Abelian category of sheaves with values in Abelian groups on the topological space $Z(\mathbb{C})$ of points of $Z$ with values in $\mathbb{C}$ with respect to $\eta$. The functors $R_{\eta, Z}$ are symmetric monoidal [Ayo10, Lemme 2.2]. According to [Ayo10, Proposition 2.4, Théorèmes 3.4 and 3.7], they commute with the functors $f^{*}, f_{*}, f_{!}$, $f^{!}$provided that the latter are applied to constructible objects (note that commutativity holds without this restriction for the two functors $f^{*}$ and $f_{!}$). In particular, they commute with Tate twists. In [CD12, Example 17.1.7], it is shown how to obtain from the $R_{\eta, Z}$ a family of exact functors with analogous properties, which we denote by the same symbols

$$
R_{\eta, Z}: \mathrm{DM}_{\mathrm{E}, c}(Z) \longrightarrow D_{c}^{b}(Z),
$$

where the right-hand side denotes the full triangulated sub-category of $D(Z)$ of classes of bounded complexes with constructible cohomology objects. The construction can be imitated to obtain $F$-linear versions of the Betti realization. Composing with the base change via Spec $k \hookrightarrow \mathbb{B}$, we finally obtain a family of exact tensor functors

$$
R_{\eta, X}: \mathrm{DM}_{\mathrm{Б}, c}(X)_{F} \longrightarrow D_{c}^{b}\left(X_{k}\right)_{F},
$$

still referred to as the Betti realization and indexed by schemes $X$ whose base change $X_{k}:=$ $X \times_{\mathbb{B}}$ Spec $k$ is quasi-projective over $k$. The functors $R_{\eta, X}$ are symmetric monoidal; in particular, they respect the unit objects. They commute with the functors $f^{*}, f_{*}, f_{!}, f^{!}$since Spec $k \hookrightarrow \mathbb{B}$ is a projective limit of open immersions (use [CD12, Proposition 14.3.1]),

Case 2: $k$ is of characteristic zero, and $\ell$ is a prime. The $\ell$-adic realization is defined in [CD16, Section 7.2, see in particular Remarque 7.2.25]. It is a family of covariant exact functors

$$
R_{\ell, Z}: \operatorname{DM}_{\mathrm{L}, c}(Z) \longrightarrow D_{c}^{b}(Z),
$$

indexed by $k$-schemes $Z$ of finite dimension. Its target is the bounded "derived category" of

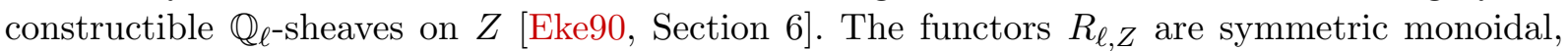
and they commute with the functors $f^{*}, f_{*}, f_{!}, f^{!}$[CD16, Théorème 7.2.24]. In particular, they commute with Tate twists. The construction can be imitated to obtain $F$-linear versions of the $\ell$-adic realization. Composing with the base change via Spec $k \hookrightarrow \mathbb{B}$, we finally obtain a family of exact tensor functors

$$
R_{\ell, X}: \mathrm{DM}_{\mathrm{b}, c}(X)_{F} \longrightarrow D_{c}^{b}\left(X_{k}\right)_{F},
$$

still referred to as the $\ell$-adic realization. The $R_{\ell, X}$ are symmetric monoidal, and they commute with the functors $f^{*}, f_{*}, f_{!}, f^{!}$.

In both settings, the categories $D_{c}^{b}\left(X_{k}\right)_{F}$ are equipped with a perverse $t$-structure; write $D^{t=0}\left(X_{k}\right)$ for its heart, $H^{n}: D_{c}^{b}\left(X_{k}\right)_{F} \rightarrow D^{t=0}\left(X_{k}\right)$, for $n \in \mathbb{Z}$, for the cohomology functors, and

$$
H^{*} R_{X}:=\left(H^{n} R_{X}\right)_{n \in \mathbb{Z}}: \operatorname{DM}_{\mathrm{L}, c}(X)_{F} \longrightarrow \operatorname{Gr}_{\mathbb{Z}} D^{t=0}\left(X_{k}\right)
$$

for the collection of all cohomology functors, preceded by the realization $R_{X}$. Here, we denote by $\mathrm{Gr}_{\mathbb{Z}} D^{t=0}\left(X_{k}\right)$ the $\mathbb{Z}$-graded category associated with the heart $D^{t=0}\left(X_{k}\right)$. We shall often refer to $H^{*} R_{X}$ as the cohomological realization.

Remark 4.1. If $k$ is finitely generated over $\mathbb{Q}$, then the $\ell$-adic realization is a realization "with weights" as the action of local Frobenii allows for a notion of purity. By contrast, there are no intrinsic weights on the target category of the Betti realization. The ideal solution would be to 


\section{J. Wildeshaus}

replace it by the Hodge-theoretic realization

$$
R_{\mathbf{H}, \eta, X}: \operatorname{DM}_{\mathrm{E}, c}(X)_{F} \longrightarrow D^{b}\left(\mathbf{M H M}_{\mathbb{Q}}\left(X \times{ }_{\eta} \mathbf{S p e c} \mathbb{C}\right) \otimes_{\mathbb{Q}} F\right)
$$

to the bounded derived category of algebraic mixed Hodge modules on $X \times{ }_{\eta}$ Spec $\mathbb{C}$ [Sai90, Section 4.2].

Let $R_{\bullet}: \mathrm{DM}_{\mathrm{E}, c}(\bullet)_{F} \longrightarrow D_{c}^{b}\left(\bullet_{k}\right)_{F}$ be one of the two realizations considered above (Betti or $\ell$-adic).

Proposition 4.2. Let $Y=Y(\Phi)=\coprod_{\varphi \in \Phi} Y_{\varphi}$ be a good stratification with nilregular strata such that the generic points of all $Y_{\varphi}$ lie over $\operatorname{Spec} k \hookrightarrow \mathbb{B}$. In the context of the Betti realization, assume that $Y_{k}$ is quasi-projective over $\mathbf{S p e c} k$. Then the restriction of the cohomological realization functor on $Y$ to $\mathrm{DM}_{\mathrm{E}, c, \Phi}^{A b}(Y)_{F}$,

$$
H^{*} R_{Y}: \mathrm{DM}_{\mathrm{B}, c, \Phi}^{A b}(Y)_{F} \longrightarrow \operatorname{Gr}_{\mathbb{Z}} D^{t=0}\left(Y_{k}\right),
$$

satisfies assumptions (1), (2), (4), and (5) of Theorem 2.10. If $R_{\bullet}$ is the $\ell$-adic realization, then

$$
H^{*} R_{\ell, Y}: \mathrm{DM}_{\mathrm{E}, c, \Phi}^{A b}(Y)_{F} \longrightarrow \mathrm{Gr}_{\mathbb{Z}} D^{t=0}\left(Y_{k}\right)
$$

also satisfies assumption (3) of Theorem 2.10.

Proof. The boundedness of the weight structure on $\mathrm{DM}_{\mathrm{E}, c, \Phi}^{A b}(Y)_{F}$ is Theorem 3.6(b). By Theorem 3.10, its heart $\mathrm{CHM}_{\Phi}^{A b}(Y)_{F}$ is semi-primary and pseudo-Abelian. According to [Wil17a, Corollary 7.13] (see Lemma 3.8), the restriction of $H^{*} R_{Y}$ to $\operatorname{DM}_{\mathrm{E}, c, \Phi}^{A b}(Y)_{F}$ maps the radical to zero.

Thus, assumptions (1), (2), and (4) of Theorem 2.10 are met. Let us check assumption (5); that is, let us show that the zero motive is the only Chow motive in $\operatorname{CHM}_{\Phi}^{A b}(Y)_{F}$ whose realization is zero.

First, given Theorem 3.11, we may assume that $\mathbb{B}$ equals the generic point $\mathbf{S p e c} k$. Thus, we have $Y_{k}=Y$.

Second, by Definition 3.5(b) and by [Wil17a, Corollary 4.10(b)], the triangulated category $\mathrm{DM}_{\mathrm{E}, c, \Phi}^{A b}(Y)_{F}$ is obtained by successive gluing over the strata $Y_{\varphi}$ of triangulated sub-categories $\mathrm{DM}_{\mathrm{E}, c}^{A b}\left(Y_{\varphi}\right)_{F}^{\prime}$ of $\mathrm{DM}_{\mathrm{E}, c}^{A b}\left(Y_{\varphi}\right)_{F}$. The $\operatorname{DM}_{\mathrm{E}, c}^{A b}\left(Y_{\varphi}\right)_{F}^{\prime}$ inherit the weight structure (Theorem 3.6) from $\mathrm{DM}_{\mathrm{E}, c}^{A b}\left(Y_{\varphi}\right)_{F}$, and according to Theorem 3.10, their hearts are semi-primary and pseudo-Abelian. Thus, the abstract theory of intermediate extensions can be applied: according to [Wil17a, Summary 2.12], any object of $\operatorname{CHM}_{\Phi}^{A b}(Y)_{F}$ is a direct sum of Chow motives of the form $j_{\varphi, ! *} M_{\varphi}$, for certain Chow motives $M \in \mathrm{CHM}^{A b}\left(Y_{\varphi}\right)_{F}$. Here, $j_{\varphi, ! *}$ denotes the intermediate extension [Wil17a, Definition 2.10] associated with the immersion $j_{\varphi}: Y_{\varphi} \hookrightarrow Y$. If the cohomological realization $H^{*} R_{Y_{\varphi}}(M) \in \mathrm{Gr}_{\mathbb{Z}} D^{t=0}\left(Y_{\varphi}\right)$ is zero, then so are the cohomological realizations of all $j_{\varphi, ! *} M_{\varphi}$, hence of all $M_{\varphi}$ since $R_{\bullet}$ is compatible with inverse images. Thus, we may assume that the stratification $\Phi$ consists of a single (nilregular) stratum: $Y(\Phi)=Y_{\varphi}$.

According to Lemma 3.8, [Wil17a, Proposition 5.5], and Proposition 3.9, any Chow motive $M$ in $\mathrm{CHM}^{A b}\left(Y_{\varphi}\right)_{F}$ is smooth and finite-dimensional. The same is therefore true for its pull-back $M_{\xi}$ to any generic point $\xi$ of $Y_{\varphi}$.

So if we assume the cohomological realization $H^{*} R_{Y_{\varphi}}(M) \in \mathrm{Gr}_{\mathbb{Z}} D^{t=0}\left(Y_{\varphi}\right)$ to be zero, then the realization of any $M_{\xi}$ is zero, again since $R_{\bullet}$ is compatible with inverse images. Therefore [Kim05, Corollary 7.3], all $M_{\xi}$ are zero. But according to [O'Su11, end of Section 5.1, pp. 54-55], this implies that $M$ is zero. 


\section{WEIGHTS AND CONSERVATIVITY}

If $R_{Y}$ is the $\ell$-adic realization, then hypothesis (3) of Theorem 2.10 is also met; that is, the morphisms in the image of $H^{*} R_{\ell, Y}$ are strict with respect to the weight filtration [Bon15, Theorem 2.5.4(II)(1), Proposition 1.3.2(II)(2)]. Note that $Y_{k}$ is of finite type over $k$, hence very reasonable in the sense of [Bon15, Definition 2.1.1(4)].

Here is our second result on conservativity in the motivic context.

THEOREM 4.3. (a) Let $Y=Y(\Phi)=\coprod_{\varphi \in \Phi} Y_{\varphi}$ be a good stratification with nilregular strata, such that the generic points of all $Y_{\varphi}$ lie over $\mathbf{S p e c} k \hookrightarrow \mathbb{B}$. In the context of the Betti realization, assume that $Y_{k}$ is quasi-projective over $\mathbf{S p e c} k$. Then the restriction of the realization functor on $Y$ to $\mathrm{DM}_{\mathrm{E}, c, \Phi}^{A b}(Y)_{F}$,

is conservative.

$$
R_{Y}: \mathrm{DM}_{\mathrm{E}, c, \Phi}^{A b}(Y)_{F} \longrightarrow D_{c}^{b}\left(Y_{k}\right)_{F}
$$

(b) Assume $\mathbb{B}=$ Spec $k$. Let $Y=Y(\Phi)=\coprod_{\varphi \in \Phi} Y_{\varphi}$ be a good stratification with nilregular strata. In the context of the Betti realization, assume that $Y$ is quasi-projective over Spec $k$. Then the restriction of the realization functor on $Y$ to $\mathrm{DM}_{\mathrm{b}, c, \Phi}^{A b}(Y)_{F}$,

$$
R_{Y}: \mathrm{DM}_{\mathrm{E}, c, \Phi}^{A b}(Y)_{F} \longrightarrow D_{c}^{b}(Y)_{F},
$$

is conservative.

Proof. Part (b) is a special case of part (a) (put $\mathbb{B}=\operatorname{Spec} k$ ).

For the $\ell$-adic realization, Theorem 2.10 can be applied directly, thanks to Proposition 4.2.

Let us treat the case where $R_{\bullet}$ is the Betti realization. Given our present state of knowledge, we may suppose, but do not know, the analogue of hypothesis (3) of Theorem 2.10 to hold; therefore, we need an alternative approach.

As earlier (Theorem 3.11 and its proof), we may assume that $\mathbb{B}$ equals the generic point Spec $k$ and that the stratification of $Y(\Phi)$ consists of a single (nilregular) stratum: $Y(\Phi)=Y_{\varphi}$.

Now, recall from the proof of Theorem 2.10 that hypothesis (3) is only used via Lemma 2.9. The idea therefore consists in deducing Lemma 2.9 from the little we know. By Lemma 3.8, [Wil17a, Proposition 5.5], and Proposition 3.9, the category $\mathrm{CHM}^{A b}\left(Y_{\varphi}\right)_{F}$ consists of Chow motives which are smooth over $Y_{\varphi}$. As it generates the triangulated category $\operatorname{DM}_{\mathrm{b}, c}^{A b}\left(Y_{\varphi}\right)_{F}$ (Theorem 3.6(b)), the cohomological realization of any object of the latter gives perverse sheaves which are actually local systems (up to a shift). This holds in particular for the objects $X$ and $Z$ occurring in Lemma 2.9; therefore, the effect of the cohomological realization of a morphism between them can be read off the restriction of the latter to the generic points of $Y_{\varphi}$, where comparison with the $\ell$-adic realization is available.

The third and main result on conservativity reads as follows; it generalizes the $\ell$-adic version of [Wil15, Theorem 1.13].

THEOREM 4.4. Assume $k$ to be of characteristic zero, and let $\ell$ a prime. Let $Y=Y(\Phi)=\coprod_{\varphi \in \Phi} Y_{\varphi}$ be a good stratification with nilregular strata such that the generic points of all $Y_{\varphi}$ lie over Spec $k \hookrightarrow \mathbb{B}$. Then the $\ell$-adic realization $R_{\ell, Y}$ respects and detects the weight structure on $\mathrm{DM}_{\mathrm{E}, c, \Phi}^{A b}(Y)_{F}$. More precisely, let $M \in \mathrm{DM}_{\mathrm{E}, c, \Phi}^{A b}(Y)_{F}$, and let $\alpha \leqslant \beta$ be two integers.

(a) The motive $M$ lies in the heart $\operatorname{CHM}_{\Phi}^{A b}(Y)_{F}$ of $w$ if and only if the $n$th perverse cohomology object $H^{n} R_{\ell, Y}(M) \in D^{t=0}\left(Y_{k}\right)$ of $R_{\ell, Y}(M)$ is pure of weight $n$ for all $n \in \mathbb{Z}$. 


\section{J. Wildeshaus}

(b) The motive $M$ lies in $D M_{\mathrm{b}, c, \Phi}^{A b}(Y)_{F, w \leqslant \alpha}$ if and only if $H^{n} R_{\ell, Y}(M)$ is of weights at most $n+\alpha$ for all $n \in \mathbb{Z}$.

(c) The motive $M$ lies in $D M_{\mathrm{E}, c, \Phi}^{A b}(Y)_{F, w \geqslant \beta}$ if and only if $H^{n} R_{\ell, Y}(M)$ is of weights at least $n+\beta$ for all $n \in \mathbb{Z}$.

(d) The motive $M$ is without weights $\alpha, \alpha+1, \ldots, \beta$ if and only if $H^{n} R_{\ell, Y}(M)$ is without weights $n+\alpha, n+\alpha+1, \ldots, n+\beta$ for all $n \in \mathbb{Z}$.

Proof. According to Proposition 4.2, the assumptions of Theorem 2.11 are satisfied for $\mathcal{H}=$ $H^{0} R_{\ell, Y}$.

Remark 4.5. If $k$ is finitely generated over $\mathbb{Q}$, then there is an intrinsic notion of weights on those objects of the heart $D^{t=0}\left(Y_{k}\right)$ of the perverse $t$-structure on $D_{c}^{b}\left(Y_{k}\right)_{F}$ which are in the image of the cohomological realization [Bon15, Proposition 2.5.1(II)].

In general, the weights of $H^{*} R_{\ell, Y}(M)$ are by definition those induced by the weight filtration of the functor $H^{*} R_{\ell, Y}$ as considered in the previous section (these coincide with the above when $k$ is finitely generated over $\mathbb{Q}$ ).

Remark 4.6. The analogue of Theorem 4.4 should hold for the Betti and the Hodge-theoretic realization. In the absence of the latter, and/or a general comparison statement between the Betti and the $\ell$-adic realization, the problem for the Betti realization consists in the verification of assumption (3) of Theorem 2.10 (which directly enters the proof of Theorem 2.11). Contrary to the proof of Theorem 4.3, reduction to a statement on individual strata of $Y(\Phi)$ does not seem to work.

Let us spell out the special case $\mathbb{B}=$ Spec $k$ of Theorem 4.4 .

Corollary 4.7. Let $k$ be a field of characteristic zero, $\ell$ a prime, $Y$ a scheme over $\mathbb{B}=\mathbf{S p e c} k$, and $Y=Y(\Phi)=\coprod_{\varphi \in \Phi} Y_{\varphi}$ a good stratification with nilregular strata. Let $M \in \mathrm{DM}_{\mathrm{E}, c, \Phi}^{A b}(Y)_{F}$, and let $\alpha \leqslant \beta$ be two integers.

(a) The motive $M$ lies in the heart $\operatorname{CHM}_{\Phi}^{A b}(Y)_{F}$ of $w$ if and only if the $n$th perverse cohomology object $H^{n} R_{\ell, Y}(M) \in D^{t=0}(Y)$ of $R_{\ell, Y}(M)$ is pure of weight $n$ for all $n \in \mathbb{Z}$.

(b) The motive $M$ lies in $D M_{\mathrm{b}, c, \Phi}^{A b}(Y)_{F, w \leqslant \alpha}$ if and only if $H^{n} R_{\ell, Y}(M)$ is of weights at most $n+\alpha$ for all $n \in \mathbb{Z}$.

(c) The motive $M$ lies in $D M_{\mathrm{b}, c, \Phi}^{A b}(Y)_{F, w \geqslant \beta}$ if and only if $H^{n} R_{\ell, Y}(M)$ is of weights at least $n+\beta$ for all $n \in \mathbb{Z}$.

(d) The motive $M$ is without weights $\alpha, \alpha+1, \ldots, \beta$ if and only if $H^{n} R_{\ell, Y}(M)$ is without weights $n+\alpha, n+\alpha+1, \ldots, n+\beta$ for all $n \in \mathbb{Z}$.

\section{ACKNOWLEDGEMENTS}

I wish to thank F. Déglise for useful discussions and comments, and F. Lecomte and A. Papadopoulos for the invitation to the 95e Rencontre entre mathématiciens et physiciens théoriciens: "Géométrie, arithmétique et physique: autour des motifs" (IRMA Strasbourg, May 2015), where the main results of the present article were presented. The observations of the referee helped to improve its legibility. 


\section{WEIGHTS AND CONSERVATIVITY}

\section{REFERENCES}

AK02 Y. André and B. Kahn, Nilpotence, radicaux et structures monoïdales (avec un appendice de P. O'Sullivan), Rend. Sem. Mat. Univ. Padova 108 (2002), 107-291; http://www. numdam.org/ item?id=RSMUP_2002_108__107_0; Erratum, Rend. Sem. Mat. Univ. Padova 113 (2005), 125128; http://www . numdam.org/item?id=RSMUP_2005_-113_-125_0.

Ayo07 J. Ayoub, Les six opérations de Grothendieck et le formalisme des cycles évanescents dans le monde motivique. II, Astérisque 315 (2007).

Ayo10 - Note sur les opérations de Grothendieck et la réalisation de Betti, J. Inst. Math. Jussieu 9 (2010), no. 2, 225-263; https://doi.org/10.1017/S1474748009000127.

Bon10 M.V. Bondarko, Weight structures vs. t-structures; weight filtrations, spectral sequences, and complexes (for motives and in general), J. K-Theory 6 (2010), no. 3, 387-504; https://doi. org/10.1017/is010012005jkt083.

Bon11_, $\mathbb{Z}[1 / p]$-motivic resolution of singularities, Compos. Math. 147 (2011), no. 5, 1434-1446; https://doi.org/10.1112/S0010437X11005410.

Bon14 W Weights for relative motives: relation with mixed complexes of sheaves, Int. Math. Res. Not. 2014 (2014), no. 17, 4715-4767; https://doi.org/10.1093/imrn/rnt088.

Bon15 - Mixed motivic sheaves (and weights for them) exist if 'ordinary' mixed motives do, Compos. Math. 151 (2015), no. 5, 917-956; https://doi.org/10.1112/S0010437X14007763.

CD12 D.-C. Cisinski and F. Déglise, Triangulated categories of mixed motives, 2012, arXiv:0912.2110v3.

CD16 Étale motives, Compos. Math. 152 (2016), no. 3, 556-666; https://doi.org/10.1112/ S0010437X15007459.

Eke90 T. Ekedahl, On the adic formalism, The Grothendieck Festschrift, Vol. II, Progr. Math., vol. 87 (Birkhäuser Boston, Boston, MA, 1990), 197-218; https://doi.org/10.1007/ 978-0-8176-4575-5_4.

Héb11 D. Hébert, Structure de poids à la Bondarko sur les motifs de Beilinson, Compos. Math. 147 (2011), no. 5, 1447-1462; https://doi.org/10.1112/S0010437X11005422.

Ivo16 F. Ivorra, Perverse, Hodge and motivic realizations of étale motives, Compos. Math. 152 (2016), no. 6, 1237-1285; https://doi.org/10.1112/S0010437X15007812.

Kim05 S. I. Kimura, Chow groups are finite dimensional, in some sense, Math. Ann. 331 (2005), no. 1, 173-201; https://doi.org/10.1007/s00208-004-0577-3.

Kün94 K. Künnemann, On the Chow motive of an abelian scheme, Motives (Seattle, WA, 1991), Proc. Sympos. Pure Math., vol. 55 (U. Jannsen, S. Kleiman, and J.-P. Serre, eds)(Amer. Math. Soc., Providence, RI, 1994), 189-205.

Lev09 M. Levine, Smooth motives, in Motives and Algebraic Cycles, Fields, Inst. Commun., vol. 56 (Amer. Math. Soc., Providence, RI, 2009), 175-231.

Lev10 , Tate motives and the fundamental group, in Cycles, Motives and Shimura Varieties, Tata Inst. Fund. Res. Stud. Math., vol. 21 (Tata Inst. Fund. Res., Mumbai, 2010), 265-392.

O'Su11 P. O'Sullivan, Algebraic cycles on an abelian variety, J. reine angew. Math. 654 (2011), 1-81; https://doi.org/10.1515/CRELLE.2011.025.

Sai90 M. Saito, Mixed Hodge modules, Publ. Res. Inst. Math. Sci. 26 (1990), no. 2, 221-333; https: //doi.org/10.2977/prims/1195171082.

Wil09 J. Wildeshaus, Chow motives without projectivity, Compos. Math. 145 (2009), no. 5, 1196-1226; https://doi.org/10.1112/S0010437X0900414X.

Wil12 , Motivic intersection complex, in Regulators, Contemp. Math., vol. 571 (Amer. Math. Soc., Providence, RI, 2012), 255-276; https://doi.org/10.1090/conm/571/11332.

Wil15_, On the interior motive of certain Shimura varieties: the case of Picard surfaces, Manuscripta Math. 148 (2015), no. 3-4, 351-377; https://doi.org/10.1007/ s00229-015-0747-5. 


\section{J. WiLDESHAUS}

Willa _ Intermediate extension of Chow motives of Abelian type, Adv. Math. 305 (2017), 515600; https://doi.org/10.1016/j.aim.2016.09.032.

Wil17b - On the intersection motive of certain Shimura varieties: the case of Siegel threefolds, 2017, arXiv:1706.02743.

Jörg Wildeshaus wildesh@math.univ-paris13.fr

Université Paris 13, Sorbonne Paris Cité, LAGA, CNRS (UMR 7539), F-93430 Villetaneuse, France 